\title{
REPRESENTATION AS EPISTEMIC IDENTIFICATION
}

\author{
John Dilworth
}

\begin{abstract}
In a previous Philo article, it was shown how properties could be ontologically dispensed with via a representational analysis: to be an $X$ is to comprehensively represent all the properties of an $X$. The current paper extends that representationalist (RT) theory by explaining representation itself in parallel epistemic rather than ontological terms. On this extended RT (ERT) theory, representations of $X$, as well as the real $X$, both may be identified as providing information about $X$, whether partial or comprehensive. But that information does not match ontological, property-based analyses of $X$, so it is epistemically fundamental-hence supporting a broadly conceptualist rather than nominalist metaphysics.
\end{abstract}

In a previous Philo article, "A Representationalist Theory of Generality" (hereafter "the RT article"), ${ }^{1}$ I showed how properties could be ontologically dispensed with via use of representational concepts. The basic idea is that in familiar cases of non-conventional representation, such as a picture $A$ of Napoleon, the picture $A$ represents Napoleon in virtue of some of its properties representing some of Napoleon's properties. But such an account has an existentially quantified structure, suggesting that a universally quantified version might also be of theoretical interest. I showed how such comprehensive cases of representation-in which all of the properties of an item $X$ represent all of the properties of an $X$-could serve as an adequate nominalistic substitute for properties as realistically conceived. Thus Napoleon, though a genuine individual or particular, would not actually possess any properties, but instead he would comprehensively represent all the properties of Napoleon.

It was also shown how the apparent references to properties in these examples could be eliminated, in a world consisting of nothing but particulars. In familiar non-comprehensive representational cases such as a picture $A$ of Napoleon, particular $A$ represents the particular individual Napoleon. 
In comprehensive cases, an apparent case $A$ of a real goat is a particular that comprehensively represents $a$ particular goat $B$-but there is no such particular goat $B$. Nevertheless, this does not prevent particular $A$ from representing $B$, because it is a familiar fact about representations that there can be representations of particular items which do not exist, such as a unicorn, or Santa Claus.

Note that on this view of representation, it is always particulars that are represented. Nevertheless, as in the example just given, the represented particular need not exist. Thus two kinds of representation can be distinguished: familiar cases of external representation, in which one particular represents another actual particular (a picture of Napoleon represents Napoleon), and also cases of internal representation, in which the representational status of a particular is independent of whether or not it externally represents a particular. $^{2}$ An example is provided by pictures that represent mythological scenery and people, which items have no independent existence as particulars.

Now in the resulting representationalist (RT) theory, the concept of representation itself was taken as a primitive, and it was assumed to be an objective fact that certain objects or their parts are capable of representing other objects and their supposed properties. It was also claimed that a person who could learn to recognize which objects, or combinations of parts, represent which other objects or represented properties, could then use the language of properties-reinterpreted as a language about represented propertiesto identify the relevant objects and parts, just as well as could a language-user in a world in which there were real properties and instances of them. The current paper will concentrate on filling out this account of the objectivity and cognitive utility of representational concepts.

Nevertheless, whereas the previous RT paper presented an argument for ontological nominalism, the current paper instead concentrates on providing a broadly conceptualist and epistemic account of representational concepts themselves. Indeed, the papers are logically independent, in that even if there are some genuine properties, the previously developed representational concepts would still to be of significant value in understanding cognitive processes of recognition, identification and classification. So unqualified references to properties in the rest of the paper are either to genuine properties, or to representational substitutes of some kind.

Also, as in the previous RT article, the somewhat unwieldy term "representation" will be abbreviated, where appropriate, as "rep," which may be used in verb form (" $A$ reps/is reping/repd $B$ " for "A represents/is representing/represented $B$ ") or noun form (" $A$ is a rep of $B$ " for " $A$ is a representation of $B$," or "a rep analysis" for "a representational analysis").

\section{Ontological Identity Versus Epistemic Identification}

A useful initial way in which to distinguish ontological from epistemic issues about representation is in terms of a contrast between the concepts of iden- 
tity and identification. The identity of an item is usually explained in terms of its ontological constituents, such as a particular and various properties. However, by contrast, identification of an item is typically an epistemic, cognitive process that may or may not have ontological implications.

In what follows, as announced, I shall primarily explain representation in epistemic rather than ontological or metaphysical terms. Thus, instead of analyzing a particular object $X$ in terms of its identity-criteria-as to those properties the possession of which is necessary and sufficient for it to be $X$, and so on-I shall instead focus on the identification-criteria for that object $X$, that is, as to the conditions under which one could reasonably or justifiably claim to have identified that object $X$, or its properties.

To begin, I shall argue that issues concerning conditions for successful identification of an object $X$ are in various significant ways distinct from identity issues concerning $X$. For after all, we routinely identify all kinds of things in terms of noticed characteristics that may have little to do with the identity-conditions for those things.

For example, one might instantly identify a classic film on the basis of viewing a few seconds of it, or establish which classical symphony one is listening to from a fragment of characteristic tonal and harmonic progression heard from the second movement. But the establishment of such identifications hinges on characteristics which at most comprise only a small fraction of the necessary conditions for the identity of the movie or symphony in question, which certainly could not amount to logically sufficient identityconditions for them, and which also cannot be logically sufficient to distinguish the works one saw or heard from other works, which might also include the same film sequence or musical sequence. Thus, in general, such characteristic signs or identifying features, which epistemically justify to a reasonably high degree the identification of something as being $X$, may have only a peripheral or minor connection with $X$ 's inherent nature and identity.

The distinctness of identification from identity-criteria becomes even more striking if we consider identification cases in which primarily relational, rather than inherent, properties of an object $X$ are involved. For example, a not uncommon plot idea in detective stories is that a kidnapped and blindfolded victim might nevertheless be able to identify a specific bridge he was driven over, by the sound the car made in crossing it-the recurrence of which sound he may later use, after his release, to re-identify the bridge in question. But of course, in thus identifying the bridge he is not identifying any inherent property of it, but only making use of its relational property of causing both itself and something else, namely a motor vehicle, to emit a characteristic pattern of sound when the vehicle is driven over the bridge in question. Thus, the sound is sufficient to identify the bridge, in spite of its not depending in any direct way on the normal or inherent identity-criteria for the bridge.

In this example we see also a crucial factor pertaining to representation, namely that the identification of the bridge via the sound is an indirect identification: the bridge itself is not identified by direct observation of it, but instead the sound is identified as representing that particular bridge (rather 
than as representing some other bridge, or some other structure of the road on which the car is being driven).

But exactly what is involved in this process of indirectly identifying the referent or object of a rep? One critical element is a concurrent or prior direct identification of the representational content of that sound, which is what provides information sufficient to indirectly identify the referent. Here is a brief explanation of the role of representational or informational content in that process.

Using the "bridge sound" example again, if the sound is heard simply as a sound, having its own characteristics, then an indirect identification of its cause cannot even begin. It is only when the sound is regarded or perceived as having some representational content, or subject matter-that is, as its being indicative of, or as providing evidence of, or information about, its cause- that one can attempt to identify which particular object might be the one that the sound provides evidence about. Or, in slogan form, no representation without content: that is, an object cannot rep or indicate or point to some other object that is to be identified unless it is regarded as having content, or meaning, or a subject matter.

Thus, the process of indirectly identifying the referent or object of a rep involves the following elements. To begin with, clearly there is a semantic or referential component involved, in that one's identification of a representing object $A$ as indirectly identifying $X$ is analyzable as one's identification of the rep content of $A$ as referring or pointing to $X$ (rather than as pointing to some other thing): that is what makes the identification of the relevant object as $X$ an indirect one. But at the same time this account presupposes that one has, in so doing, directly identified something else, namely the rep content (hereafter just: content) of $A .^{3}$

This view of typical cases of rep as involving two integrally related kinds of epistemic identification, one direct and the other indirect, can potentially be generalized to apply to signs of every kind, whether natural or conventional. (See section 8 for a discussion of linguistic signs.) To be a non-conventional or natural sign of $X$, on this epistemic approach, is to be an object (or event, etc.) that provides, in some specific natural or cultural context, a reasonably justified indirect identification of the object $X$ that it represents. Then more culturally specific concepts of representation, such as that of pictorial rep or depiction, or of linguistic rep, can be specified-but which categories nevertheless are constructed using this same basic kind of analysis.

As an example of a natural sign, the characteristic sound made by the interaction of a car with a bridge, when driven over a specific bridge $X$, is a natural sign of that bridge $X$, and a suitably informed listener can hear that sound as representing or signifying bridge $X$ if she has learned to indirectly identify the bridge in question simply on the basis of hearing the sound in question as providing meaningful identifying information regarding the bridge as part of its content. Thus, in sum, for suitably informed listener $A$, sound $B$ reps or signifies bridge $X$ just in case $A$ can indirectly identify or recognize bridge $X$ as a result of her perceptually grasping the relevant con- 
tent of sound $B$. Or in a pictorial case, a picture of an actual building is directly identifiable as having that building as part of its content, which enables one to indirectly identify the building itself. ${ }^{4}$

To summarize, this section has explored typical cases of using a rep to achieve indirect identification of another item, in which perception of the content of one item $A$ is used as a means of indirectly identifying another item $B$ that is represented by $A$. For example, directly identifying a picture as being a picture of Napoleon can enable a viewer to indirectly identify its subject as being the actual person Napoleon.

\section{Veridical Identification Difficulties}

Recall that the previous RT article explained the difference between a rep of an $X$ and a real $X$ in broadly quantificational terms. Typical representational pictures are both partial - in that only some of their properties have a rep function-and incomplete, in that they rep only some properties of a real $X$. On the other hand, a supposedly real $X$ was claimed to be a comprehensive rep, in that all of its properties internally rep all of the properties of a real $X$. In this manner the apparently absolute distinction between a rep of an $X$ and a real $X$ could be dispensed with.

However, in the current, independent paper we are allowing the possibility that there might be some genuine properties, and hence a legitimate absolute distinction between reps of an $X$ versus a real $X$ in some cases. Also, even if there were no properties, there would still remain a genuine distinction between comprehensive and non-comprehensive reps of an $X$.

But once cognitive or epistemic concepts such as recognition or identification are introduced into the discussion, the following kind of problem arises. Normally concepts such as those of perception, recognition and identification are taken to be veridical concepts, in the sense that one cannot recognize or identify an item as being $X$, or an $X$, unless it $i s$ or an $X$. But if so, this would seem to preclude there being any genuine cases of recognition or identification of the contents of pictures or other non-comprehensive reps with respect to the particular items that they externally represent. For example, how could one genuinely recognize or identify Napoleon when looking at a picture of him, in virtue of the Napoleon-related content of the picture, since neither the picture itself, nor its content, is identical with the individual externally repd by the picture, namely Napoleon?

Thus, the purely ontological, quantificational account of such reps in the previous RT article does not by itself provide an explanation as to how such apparent identifications could be genuine or legitimate, since as noted above, the same problem would arise as a distinction between legitimate identifications of comprehensive reps of a particular $X$ as being $X$, versus illegitimate identifications of non-comprehensive reps of $X$ as being $X$. Thus, the specific locus of the problem is cases of external rep in which one particular reps another: since they are distinct particulars, how could one correctly be identified as being the other? 
There are at least four dimensions of this problem, two of which have been dealt with elsewhere and which hence will only be summarized here. First, the epistemic considerations presented in section 1 above show that central cases of the epistemic utility of external reps depend on directly identifying their content as a means to indirectly identifying the item that they externally represent. Hence in such cases it is clear that two distinct concepts of identification are at work, which are closely related to the distinction of internal from exernal rep.

As long as the distinct concepts of identification or recognition are not confused, no inconsistency need result. In brief, the two concepts are linked in the following manner: direct identification of the internally repd item $X$ is a necessary condition of one's being able to indirectly identify the externally repd particular $X$. But direct identification of $X$ itself either involves no rep at all (if there are properties), or it is purely a matter of comprehensive internal rep. The only problematic case is that in which one particular noncomprehensively and externally reps another, but in that case the direct versus indirect identification distinction resolves the apparent conflict. ${ }^{5}$

The second dimension of the problem is as follows. There is the closely related issue of the distinction of a real from a merely representational $X$, whether as an absolute ontological distinction, or as a comprehensive versus non-comprehensive rep distinction. This problem can be handled by a broadly functionalist, naturalistic theory of rep, which distinguishes different cognitive attitudes toward real versus merely representational cases of an $X .^{6}$

As long as the attitudes are distinct, so that no cognitive confusions of real with representational cases occur, then it is harmless to identify a rep of $X$ as being $X$. For example, one might be disposed to jump into a real swimming pool, but not disposed to jump into a picture of that same swimming pool, and hence one's identification of each as being, or as having as its content, that same swimming pool is cognitively harmless, since no practical confusion results. This solution to the real versus representational cases is also fully consistent with the previous distinction of direct versus indirect kinds of identification in the explanation of the first factor involved in the problem, as discussed above.

However, two further dimensions of the original problem yet remain, whose adequate resolution will occupy us for the remainder of this paper. They are significant because the RT theory in the previous article does not address them, while the solutions to the first two dimensions of the problem summarized above are of only peripheral help. These two further dimensions will be described in the next section.

\section{Two Extra Identification Probiems}

By way of introduction to the first of our two remaining problems, consider a series of increasingly detailed pictures of the English group of rocks or stones making up the monument Stonehenge-from a rough sketch, providing only minimal information about the monument, to a high resolution 
color photograph of it, each from the same point of view. Each picture is identifiable as being a picture of Stonehenge, and intuitively speaking, each picture in the series provides increasing amounts of information about Stonehenge, or reps more properties of Stonehenge.

However, though it is true that in some sense each picture reps more properties of Stonehenge than do previous pictures in the series, nevertheless that atomistic way of describing the reps does not fully capture their representational differences. For in identifying each picture as being a picture of Stonehenge, one does not merely identify the particular properties of Stonehenge that each represents, but one also identifies each as reping the particular item Stonehenge as having those various properties. Or, to put the point in terms of the language of properties, each reps not only increasing proportions of the properties of Stonehenge, but each also provides an increasing amount of information about what it is to be Stonehenge; i.e., concerning Stonehenge's property of being Stonehenge.

Now the original RT theory would have explained such a series in purely atomistic terms: each picture represented more properties of Stonehenge, and that is all that there is to be said. But such an atomistic account does not explain how the series of pictures may severally be identified as reping Stonehenge itself in increasing detail-that is, either the particular entity Stonehenge, or its property of being Stonehenge. Or, to put the matter in terms of the language of information, each picture provides an increasing amount of information about Stonehenge, but that information cannot be atomistically decomposed without remainder into information about particular properties of Stonehenge.

Because of the importance of the point, it will be further rephrased as follows. A general theory about the rep of properties should not automatically assume that all properties can be atomistically reduced to a few basic kinds. Many properties associated with midsized objects, such as the property of being the relevant kind of midsized object, seem to be holistic in the sense that they cannot be reduced to, or eliminated in favor of, whatever lower level properties are also possessed by the relevant objects. But if this is so, then increasingly detailed reps of those mid-sized objects will typically represent those holistic properties in increasing detail, as well as representing more of the relevant lower level properties. Yet so far we lack any theoretical apparatus to explain how a given property itself could be repd in different ways, such as in increasing detail.

Holistic considerations are thus relevant to identification of an $X$ in reps of an $X$ in two different ways. First, the property of being a (whole) $X$ is involved, rather than just the individual properties that are possessed by whole $X$ s. And second, each picture in the series provides increasing amounts of information about that same whole item $X$, rather than just providing information about specific properties of the $X$.

The second of our two remaining problems involves an extra dimension of the identification problem just stated. In place of the original series of increasingly detailed reps of Stonehenge, imagine instead another 
series, each of which provides roughly equivalent amounts of information about Stonehenge-but with each being drawn, painted, photographed etc. using different representational methods or different artistic styles. For example, one might be a fully realistic but somewhat indefinite or blurred, another might be in a vigorous impressionist style, but which conveys roughly the same information as the indefinite realistic picture, and so on. The problem now is that each picture has Stonehenge-related content that is roughly equivalent in informational content, but each nevertheless still represents Stonehenge in a different way than do the other pictures in the series. Also, to emphasize, these different ways cannot be explained in terms of representing different properties of Stonehenge, whether holistic or lower level properties, just because of their rough equivalence in informational content.

It will turn out that the two problems are interconnected (see section 7) but for the present we shall concentrate on discussing the implications of the first problem, which could be called the varying information problem. One major implication is that the previous RT method of elimination of properties was too conservative, in that its one-to-one replacement of properties with repd properties left the logical structure of the atomistic properties unchanged. But the varying information problem shows that not only do holistic properties need to be provisionally considered-which were not considered in the original RT analysis—but that a single property, even a holistic one, is too lacking in structure to account for the varying information problem.

These two kinds of mismatch in logical structure-both of extra holistic properties being provisionally required, and of their inadequacy in matching the varying informational structure of representations of them-suggest the following. Whether or not there are any properties of atomistic or holistic kinds possessed by worldly items $X$, an adequate account of the rep content of reps of $X$ must provide an independent account of the informational content of such reps. ${ }^{7}$ To the extent that the structures of such information match those of putative properties, an RT-style elimination of those properties could be provided. But in the case of any mismatches, it is necessary to recognize the epistemic autonomy of such informational concepts from ontological property concepts. There must be legitimate roles played by the information in rep contents in a cognitive system, which roles cannot be reduced to the role of those contents in being about supposed worldly properties.

\section{Information About an $X$}

In more detail, the suggestion is that a concept of information about an $X$ needs to be introduced. Real $X \mathrm{~s}$ (if any) supply comprehensive information about an $X$, whereas reps of an $X$ provide only partial or non-comprehensive information about an $X$. But cases of both kinds can be legitimately identified as providing some information about an $X$, and hence as being veridical identifications, as long as the information provided is correct. (Thus, to this extent, an informational approach provides another way to 
solve or dissolve concerns about veridical identification of non-comprehensive reps, as discussed in section 2).

Hence both reps of an $X$, and $\mathrm{X}$ itself, may be regarded as falling on a single epistemic identificatory scale, of items that are, in a suitably broad sense, identifiable as $X$-which scale at the top, comprehensive end includes a real $X$, since that $\mathrm{X}$ itself may be identified as $X$. So, from the epistemic point of view of cognitive processing of information, a real $X$ and reps of $X$ could be regarded as being on a par: all of them are grist for the same internal cognitive identifying mechanisms with respect to processing varying amounts of information about that $X$.

But what kind of identificatory scale is involved here? Intuitively, it seems correct to describe the limiting case, as above, as one of comprehensive identification. Thus in the limiting case the concepts of comprehensive identification of an $X$, and of the identity of an $X$, apparently could be regarded as providing roughly equivalent epistemic versus ontological ways of describing the same facts about $X$-namely, identifying all of the information about an $X$, and specifying all of the properties of that same $X$ (though see the next section for a caveat).

However, this does not mean that the non-comprehensive representational cases permit any such equivalence, for as argued in section 3 using the Stonehenge example, information about an item $X$ is holistic in ways in which properties—or their representational substitutes as provided by the RT theory-are not. In view of this fact, a useful theoretical move at this stage is as follows. Since each picture in the series provides, in virtue of its $X$-related representational content, increasing amounts of information about $X$, and since that information is also what enable a viewer of the picture to recognize or identify the picture as being a picture of $X$, we may postulate that the representational content of each picture of $X$ consists of some $X$-related informational structures (ISs), perception of which prompts identification of the picture as a picture of $X .^{8}$

The relevant ISs might be holistic or relational in several ways. For example, to cite a new kind of case, the shape Si of one particular stone in Stonehenge would be repd in a picture $P$ in virtue of $P$ 's representational content containing an IS IS(Si), which may provide information not only about the shape $\mathrm{Si}$, but also comparative information about the relative size of the stone having that shape $\mathrm{Si}$ in relation to the shapes of the other stones, how that stone relates to the monument as a whole, and so on.

To be sure, a perceiver of picture $P$ may or may not, in identifying the shape of that particular stone in the picture, cognitively extract all of the information available in structure $\mathrm{IS}(\mathrm{Si})$, but pictures and other reps only count as reps at all in virtue of their being capable of supplying such information about a worldly item $X$ and its parts to perceivers of them. Indeed, this guaranteed epistemic accessibility of any informational structure contrasts with the merely contingent accessibility of an ontological property, whose instantiation by an item may or may not be discoverable by us.

To summarize, the main differences of informational structures (ISs) 
from properties uncovered so far are as follows. Either there are many different $X$-related holistic IS types, one for each of the types of reps involving different amounts of information about $X$, or the identity of a single $X$ related holistic IS persists through changes in the amount of information provided by the structure. For example, the many ISs, or single changing IS, corresponding to the property of being Stonehenge is part of the content of various reps of Stonehenge, some of which provide more information about Stonehenge than others. Whereas by contrast, presumably there is only a single, immutable property of being Stonehenge as viewed at a particular time from a particular position, or a single perspectival state of the object, so that the single property or object state does not match either conception of the relevant informational structure(s).

As to which conception of holistic $X$-related ISs is correct, a plausible view is that both are legitimate, each serving differing cognitive purposes. As pointed out by Millikan, ${ }^{9}$ there is a "consumer" aspect to representational content, such that what the content of a rep counts as being depends on the cognitive use to which that representation is put. When the cognitive emphasis is on its being the same whole object or property that the content is about, the identity-through-change conception is appropriate. But when instead the emphasis is on the differing amounts of information, the multiple IS conception is more appropriate. Or alternatively, a genus-species conception of their relations could be adopted: information about a particular object $X$ is the genus, and each specific level of information about $X$ is a species of that same genus. On that alternative conception, the interestrelativity of content would be a matter of cognitive interest in the genus rather than the species, or vice-versa.

\section{A Broader Issue: Generality Versus Particularity}

At this point, it is appropriate to raise a broader issue. The original RT theory explained the generality of properties, for example sortal properties, in terms of each individual $X$ of the relevant kind $Y$ comprehensively representing $a Y$. It also explained the numerical distinctness of particular $X \mathrm{~s}$ of kind $\mathrm{Y}$ in terms of each comprehensively representing a numerically distinct particular Y. However, it might be thought that this account does not fully explain the concept of what it is to be a particular object or individual $X$, for particular objects can be classified in indefinitely many ways, so that even comprehensive representation by an individual $X$ of some particular of kind $Y$ might be thought to underdetermine the particularity or identity of that object $X$. (There are also essentialist issues, of the kind raised by Quine,$^{10}$ as to whether explaining the individuality of $X$ in terms of its being a $Y$ invidiously classifies it as essentially a $Y$ but not essentially a $Z$, for some other sortal $Z$ that also classifies $X$ ). Or, to put the issue in terms of properties, the RT account might seem not to adequately address the issue of X's property of being that particular individual X, ${ }^{11}$ independently of ways in which it might be classified. So, either the brute particularity of $X$ isn't fully explained, or 
in terms of the language of properties, $X$ 's property of being that particular object $X$ isn't fully explained.

Now to be fair to the RT theory, it does have at least a partial answer to these accusations. First, as to brute particularity, if there are no properties then the difference of one particular from another can be taken as being primitive. Second, since the RT view explains what it is for a particular $X$ to be a real $Y$ in terms of comprehensive rep of all of the properties of a $Y$, then all possible classifications of the particular $X$ are automatically included in this account. Nevertheless it remains true that the issue of the status of $X$ 's putative uniquely particularizing property of being that particular individual $\mathrm{X}$-or of representational substitutes for it—is not specifically addressed by the RT account.

But now, with the aid of the current epistemic informational approach, that issue can be addressed. The concept initially developed in the previous section of all of the information about a particular individual $\mathrm{X}$, such that $X$ itself comprehensively reps all of that information, while other reps of $X$ noncomprehensively rep some of that information about $X$, does not depend on an analysis specifically couched in terms of a particular property-related sortal term. Thus, the current account is doubly immune to the accusation that the particularity of $X$ is slighted, as possibly it was to some extent in the original RT analysis. It is immune, first because no particular sortal is invoked in the current analysis, and second, because the issue about the particularity of $X$ itself is fully addressed, in that information about $X$ itself, or its holistic property of being that particular individual $X$, is specifically recognized as being distinct from information about other properties of $X$.

Now if we considered only $X$ 's own comprehensive internal rep of all of the information about itself, the claim that the information includes information about its holistic particularizing property of being $X$ might seem to be a mere stipulation or trick. But what gives legitimacy to that analysis is the fact that clearly there can be many non-comprehensive external reps of $X$, which include information uniquely or holistically about $X$ itself, and yet which do so in various different, informationally non-equivalent ways. Or in other words, our legitimate cognitive need to fully grasp the particularity of an object $X$ shows itself in the fact of our having potentially many different reps of that unique particularity of $X$, each of which supplies in its own way different amounts (and possibly different kinds) of information about what it is to be that particular item $X$.

This point remains valid even if there are properties, and even if some form of the Leibnizian principle of the identity of indiscernibles is true for some particulars (whose truth normally would be assumed to be incompatible with a particular having a thisness). The particular would still remain more than the properties it instantiates, because it would still comprehensively rep all of the information about itself, ${ }^{12}$ including holistic information about what it is for it to be that particular, and also non-comprehensive reps of that particular would continue to be relevant as discussed above.

The current analysis is also important for a broader metaphysical rea- 
son. It might be thought that the universal/particular distinction is a metaphysically unavoidable one, so that any attempt to eliminate universals in favor of particulars, as the RT theory attempted to do, is bound to end in failure. ${ }^{13}$ However, the current informational analysis of particularity shows that a form of the universal/particular distinction lives on in representational form, in that turns out that a full cognitive understanding of what it is to be a unique particular-even in a world where there were no properties or universals - could be achieved via the possibility of a multiplicity of distinct reps of that same individual. Thus, in this manner the concepts of universal and particular would remain as irreplaceable correlatives, even if universals were ontologically eliminated.

\section{The Independence of Informational from Property Concepts}

The position we have arrived at so far is a broadly conceptualist one. The original RT article eliminated properties of particular objects $X$ via an analysis in terms of their comprehensive internal rep of an $X$. In this paper the related concept of non-comprehensive external rep is being explained in broadly epistemic, informational terms, via an analysis of the cognitive processes involved in identification of the representational content of public representations, such as pictures, of particular worldly items $X$. A crucial point has been the provision of evidence that holistic informational content about a particular item $X$ cannot be explained in terms of a property of $X$, even of the special "thisness" kind, such as $X$ 's putative property of being that particular object $X$. Nor could the brute fact of the unique objecthood of $X$, divorced from property considerations, explain the possibility of varying kinds of holistic information about $X$ occurring in reps of $X$ providing different amounts of information about $X$.

Further evidence along the same lines will now be provided. To begin, it might be thought that $X$ 's "thisness" property, of $X$ being the particular object that it is, is a special case, which could be bracketed, or otherwise worked around, so as to restore the simpler RT account of rep content. However, this is not so, for structurally the same informational points would apply to any non-minimal visible part of $X$ as well. For example, each of the stones making up Stonehenge is such that it has the holistic property of being that particular stone, but each stone is equally representable in different informational ways, just as Stonehenge itself is. And the same goes for all of the non-minimal visible parts of the stones, that is, those parts, whether separable or inseparable, which can be seen to have parts themselves. All of them have the holistic property of being that particular chunk of matter-whether or not considered as being part of Stonehenge-which property is irreducible to the properties of their own parts, and all of them are representable in different informational ways.

A clinching point can also be provided, which is independent of holistic considerations. Certain kinds of reps, such as uniformly blurred ones, are such that all of the properties of $X$, whether holistic or not, are represented 
in informationally indeterminate ways, which hence differ from how nonblurry reps would rep all of those properties. So, informationally varied ways of reping any and all properties of any particular item X must be ubiquitous in possible reps of $X$.

Now at this point, some care is needed. We still wish to preserve the legitimate sense in which non-conventional reps of some object $X$, such as pictures of $X$, do indeed veridically externally rep $X$ and its properties, whatever may be the correct metaphysical account of objects and properties. For it is generally assumed that at least some reps of worldly particulars $X$ are veridical or correct. But at the same time, we also wish to accurately describe and explain the cognitive facts about the noted mismatches between the varying kinds of information provided by reps of an $X$, and the properties of the $X$ itself, however those properties are construed. What is needed is a broader theory of representation that can restore consistency to these apparently conflicting considerations. One such theory will now be outlined, in this and the following section.

To begin, consider again a uniformly blurred rep $A$ of some object such as Stonehenge, such as an out-of-focus photograph of it. Now on a simplistic resemblance theory of representation, since all of picture $A$ 's $X$-related content is blurred, but no part or property of Stonehenge is blurred, then $A$ completely fails to rep Stonehenge or any of its properties. Hence, $A$ could not be, or be legitimately identified as, a veridical or correct rep of Stonehenge.

However, clearly such a simplistic resemblance theory is totally inadequate. ${ }^{14}$ Though blurred picture A does not provide fully detailed information about Stonehenge, it does provide some information about it, which information is correct as far as it goes. For example, the rough configuration or arrangement of the stones, as repd in picture $A$, is indeed roughly as $A$ reps it to be. So, $A$ does not supply any misleading information about the configuration of stones in Stonehenge, and so to that extent $A$ does veridically represent Stonehenge and some of its properties.

Nevertheless, since the actual stones in Stonehenge are usually assumed to be in a precise rather than a rough configuration, the rough configuration repd by picture $A$ is not an actual relational property of Stonehenge. Approximately correct rep of properties of $X$ is not correct rep of approximate properties of $X$, because there could not be any approximate properties of anything. So, the mismatch between informational content and properties of $X$ persists-and of course, any rep $Y$ of an actual object $X$, other than an exact replica of $X$, would be blurred or indeterminate in its content at some microscope level of detail. So, the problem remains a pervasive one for reps in general. ${ }^{15}$

In schematic form, my initial suggested resolution of the problem is as follows. The previous RT theory showed how to ontologically eliminate properties. This paper is engaged in showing, in effect, that cognitive uses of public, non-comprehensive external reps, which are fundamental and pervasive in perception and cognition, are to be understood in terms of sui generis informational concepts, which only loosely correspond to traditional 
realist conceptions of the actual properties of objects, whether or not there are any such properties.

As a consequence, one natural initial view is that a concept of a veridical non-conventional rep of $X$ needs to be reinterpreted as a concept of a nonmisleading rep of $X$, that is, one such that the specific level of information about $X$ that it provides is not misleading in relation to typical cognitive uses that would be made of that information in studying $X$, or in interacting with it. For example, a bank photograph of a suspect committing a crime might be accurate enough to enable the actual perpetrator $P$ to be identified on the basis of it. In such a case, not only does the photograph rep person $P$ in virtue of his causal link to the photograph, but it also non-misleadingly reps $P$ in virtue of its facilitating correct identification of $P$ by the police. On the current view, there might be no more to its usually assumed "veridical" status than that.

An important issue arising from this view is of the relation of such broadly pictorial kinds of veridical rep to conventional linguistic reps that express propositions. A central concern could be expressed as follows. If veridicality for pictures is non-misleadingness, rather than correspondence to the properties (or representationally eliminated properties) possessed by objects, would a similar non-correspondence analysis of propositional truth also be required, or do linguistic reps work in fundamentally different ways? At this stage both options are open, because nothing previously said in this paper has directly addressed relevant issues such as that of the truthconditional propositional content of beliefs.

It is true that there are some significant differences between linguistic and pictorial reps, e.g. of the kinds recently described by Cummins and Poirier. ${ }^{16}$ Also, the current skepticism, both about the ontological reality of properties and about the cognitive utility of exclusively property-based concepts, does not imply skepticism about the reality of a mind-independent world of particulars, and so something like a traditional correspondence theory of truth would still seem to be appropriate. Both of these points would support a bifurcated view that would treat pictorial and linguistic reps separately. Nevertheless, section 8 will provisionally suggest a way of maintaining worldly correspondence for both kinds of reps, so that a common treatment for both kinds may still be possible.

\section{Two KINDS of RepRESENTATION}

This section continues the presentation of a more comprehensive theory of representation which can explain how there can both be correct reps of an object $X$ and its apparent properties, yet which reps also provide distinctively different amounts of information about object $X$, in ways that in no case precisely match $X$ 's apparent properties. It has already been explained, in outline form, how veridicality for such reps of $X$ could initially be re-conceptualized in terms of the cognitive non-misleadingness of information provided by such reps, rather than in terms of each rep having properties that match properties of $X$. 
But it still remains to provide an improved theory of rep that goes beyond the simplistic resemblance view rejected in the previous section. Recall that on such a resemblance view, since all of a blurred picture $A$ 's $X$ related content is blurred, but no part or property of Stonehenge is blurred, then $A$ completely fails to rep Stonehenge or any of its properties. As also pointed out, a more adequate theory should explain how A can provide some information about $X$ without its having to be completely specific information about $X$.

A key to providing a more adequate theory is as follows. In some way the blurred or soft-focus aspects of the $\operatorname{rep} A$ of $X$ need to be conceptually separated from the non-specific information about $X$ provided by $A$. The blurred appearance of each area of picture $A$ does not itself have a role in representing information about $X$. Instead, it is a broadly contextual or aspectual feature of picture $A$ that is independent of $A$ 's $X$-related content, since reps of any items whatsoever could have a blurred appearance.

At this point it is now possible to re-introduce the second problem from section 3, namely that even reps of $X$ which are approximately equivalent in amounts of $X$-related content may nevertheless diverge widely in stylistic features, including the medium used (e.g., drawing, painting, photography) plus whether the artist employed impressionist, expressionist, realistic etc. styles in presenting the $X$-related information. Both the main amounts-ofinformation problem and this broader stylistic problem may be resolved by distinguishing broadly contextual aspects of representations $A$, which are independent of the world-related content of $A$, from the worldly-contentrelated aspects of $A$, which are independent of the style. (Artistic style is one species of contextual or aspectual information about a rep).

A simple perceptual analogy can help to clarify the distinction. In misty conditions, a familiar object such as a house $X$ can look hazy or blurred because of the mist. But since this is perception of a real house $X$, it is taken for granted that the blurred appearance of the house does not imply that viewers of the house are seeing a blurred house. Instead, though the misty conditions contextually reduce the amount of house-related information about $X$ available to perceivers of it, perceivers automatically compensate for the misty conditions by cognitively distinguishing the mist-related information from the house-related perceptual information about $X$. My claim is that the same, or closely similar, cognitive mechanisms are at work in perception of reps of an $X$ as well. In both real and representational cases, two levels or kinds of perceptual representation are involved, with two distinct kinds of content corresponding to the distinction between contextual or aspectual factors versus object-related factors. ${ }^{17}$

Parenthetically, this "double content" kind of representational theory can also provide a convincing account of the nature of photographs and other visual artworks. Such artworks are the stylistic content represented by physical artifacts such as copies of a photograph, and that stylistic content in turn has a world-related content in virtue of its representing some worldly subject matter. ${ }^{18}$ 
We can now resolve our problem as to how widely different kinds of reps of an item $X$, such as Stonehenge, involving different kinds of aspectual or stylistic content and providing different amounts of information about Stonehenge, can nevertheless all be providing holistic information about Stonehenge itself. It is possible because perception of objects $\mathrm{X}$ in general involves two levels of representation or content, so that perception of reps of a particular object $X$ re-uses the same two-level representational mechanisms to separate out the aspectual from the object-related content.

However, this process is inevitably a holistic one, in that the original low level perceptual data, e.g., as acquired via perception in misty conditions, involves a thoroughly intermixed form of mist-related and object-related data. So, the cognitive separation of the components is an approximate, epistemically imperfect process that can provide no more than incomplete holistic information about the relevant worldly item $X$. That, in a nutshell, is the reason why there must be a mismatch between the ontological categories of objects and properties on the one hand, and epistemic categories associated with representational perceptual information on the other hand.

Nevertheless, the close association between cases of normal perception of non-representational objects, and perception of reps of other objects, ensures that the objectivity of normal perception also carries over to perception of reps. Though holistically informational, reps of items $X$ represent the relevant items $X$ just as objectively as normal perception reps its objects. (Which is not to deny that misrepresentations can occur in both cases). ${ }^{19}$

Hence we have, at least in outline form, achieved our primary goal of explaining representation in broadly epistemic, informational terms, while also preserving the objectivity of rep, and its close connection with epistemic processes of identification of items, whether in real or rep cases. The current double content account of rep is also fully consistent with the previous RT article, as well as improving on its results in various ways. (The resulting joint theory may be described as the Extended RT or ERT theory).

\section{KindS OF CORRESPONDENCE, AND LINGUISTIC REPRESENTATIONS}

This section is more speculative and open-ended than the previous discussions, but the paper would be incomplete without it. To begin, recall that in section 6 an initial view was presented, according to which the veridicality of pictures and other non-conventional reps is to be explained in terms of their providing non-misleading information about an object $X$, rather than in terms of their correspondence to the properties of $X$-since they fail to match, or correspond to, the properties of $X$. However, that initial view could simply be the result of a too limited or too conservative view about objects and properties. For even if there are properties of $X$ to which reps of $\mathrm{X}$ fail to correspond, it must not be forgotten that, according to the present theory, object $X$ itself comprehensively reps all of the information about $X$.

So, veridicality for reps of $X$ might instead be explained in terms of informational correspondence, or informational inclusion, as follows. A rep $A$ of 
$X$ is informationally veridical just in case all of the information it provides about $X$ is included in the information that $X$ comprehensively provides about itself to perceivers of it. Nevertheless, so far there is nothing to prevent us from maintaining both kinds of veridicality simultaneously. With respect to the properties of $X$, if there are any, property-based veridical rep of $X$ 's actual properties is non-misleading rep of them, whereas with respect to the information about $X$ provided by reps of $X$, veridical rep of $X$ is informational correspondence. Nevertheless, for any particular $X$ that has no genuine properties, then informational correspondence for reps of it would be the only applicable veridicality relation.

As for the previous project of the RT article in eliminating properties, now two distinct kinds of elimination may be distinguished. Conforming eliminations of a property $F$, if any, would be those in which the comprehensive representation by object $X$ of an object having property $F$ is such that part of the comprehensive information provided by $X$ conforms to our pre-theoretical concept or understanding of the relevant property. (The RT article only dealt with such conforming eliminations). Non-conforming eliminations, if any, would be those for which no such conforming relation holds. (Example: if an exact property were eliminated in favor of some inexact information, then that would be a non-conforming elimination).

Now the issue of conventional linguistic representations will be discussed. The upshot of the discussion above for non-conventional reps such as pictures is that an informationally based correspondence relation is available, whether or not there are any genuine properties, and whether or not any property eliminations are conforming. Also, even if there are some genuine properties-veridical rep of which requires provision of non-misleading information about them-there is no prima facie reason why conventional linguistic reps should not provide such information just as well as non-conventional reps. So, there are no initial roadblocks in the way of extending the results of the present paper to apply to linguistic reps also.

Here are three further arguments in favor of such an extension. First, both normal perception of a worldly item $X$, and pictures of $X$, provide only inexact information about $X$. But then one would expect that linguistic reps of $X$ would similarly only provide inexact information about $X$. So, prima facie, the same veridicality issues discussed for pictures etc. should also apply to issues concerning the truth of propositions expressed by such linguistic reps of $X$. Second, in spite of the significant differences between pictures and linguistic sentences (see the end of section 6), it is generally possible to linguistically describe the informational content of a picture to any desired level of detail. Also, these descriptions could include descriptions of the inexact information provided by the picture about $X$. But presumably those descriptions have as their content some subset of the same inexact content as the pictorial content that they describe. So again, similar correspondence structures should apply to both pictorial and linguistic description. And third, Millikan ${ }^{20}$ provides much evidence that identification of items through perception of language about them works in much the same 
way as perception of the items themselves, hence demonstrating a structure parallel to that shown here for perception of pictorial reps of an $X$ versus perception of $X$ itself.

\section{BROADER HORIzONS}

The discussion in this paper will now briefly be situated in a broader context. There are perennial issues raised in the realism versus antirealism debates ${ }^{21}$ about how our representations of the world relate to the world itself. Realists claim that we are able to mentally represent a mind-independent reality, whereas antirealists deny this, via such methods as attempting to undermine the representationalist structures assumed by the realist. The current paper could be viewed as arguing for an intermediate position in that debate. Though it assumes, with the realist, that worldly particulars exist independently of our representational cognition of them, nevertheless the concept of informational content developed here is a concept that is both mind-dependent and world-dependent. Whereas the previous RT article rejected properties on thoroughly realist ontological grounds, the current paper argues that the representationalist replacement for any such rejected properties, namely information about the world, must be understood in fundamentally epistemic rather than ontological ways.

Or, using as an analogy the hypothesized observation-dependence of quantum-mechanical phenomena on observers of them, ${ }^{22}$ the current paper in effect argues that we cannot separate objective information about the world-which we identify via use of perceptual, pictorial or linguistic representations-from our own epistemic activities of perceptually identifying and representing that information. On the account given here, information is not—as it is in the "informational semantics" of Dretske, Fodor et al ${ }^{23}$ - simply an instrumental way to learn about the actual, mind-independent properties of worldly items. Instead, information is, at the most fundamental level, a joint product of the world and our epistemic interactions with the world.

Nevertheless, the current view remains a moderate or hybrid one in another respect. It has not been denied that there may be some objective, mind-independent properties conforming to the traditional realist understanding of them. Instead it has been argued that if there are any such, a correspondence theory of truth for references to such entities must adjust itself to the informational realities of our epistemic situation via a concept of correspondence as provision of non-misleading information about such entities. (Thus we could both have our realist cake, and eat it informationally too).

As for how the current epistemic view relates to strong realist views such as physicalism, a natural employment of the current hybrid view of properties in support of a physicalist ontology would be as follows. The fundamental particles etc. and their properties, as postulated by our best science, may actually exist (subject to revisions by ongoing science), but the supposed macroscopic properties of midsized objects may be eliminated using the representational methods developed in the current extended RT (ERT) theory. 
An advantage of such a physicalism-friendly, two-tier picture of reality is that it would sidestep, for midsized objects, the apparently intractable conflicts between realism and antirealism with respect to them. For the representational elimination of their properties would remain compatible with a robust realism about the underlying physical reality, hence giving both realists and antirealists some of what each wants.

Western Michigan University

\section{NOTES}

1. "A Representationalist Theory of Generality," Philo vol. 6, no. 1 (Fall-Winter 2003): 216-234. (This and other articles cited are available from the author's Web site at http://homepages.wmich.edu/ dilworth/Index.html.)

2. For more details see my article "Internal Versus External Representation," The Journal of Aesthetics and Art Criticism vol. 62, no. 1 (Winter 2004): 23-36. details.

3. Again see my article "Internal Versus External Representation" for further

4. For a different account of natural signs claiming that all identification is direct identification, see Ruth Millikan, On Clear and Confused Ideas (Cambridge: Cambridge University Press, 2000).

5. See "Internal Versus External Representation," and for further details my book The Double Content of Art (Amherst, N.Y.: Prometheus Books, 2005).

6. See my article "The Perception of Representational Content," The British Journal of Aesthetics vol. 45, no. 4 (October 2005): 388-411.

7. For a demonstration of the inadequacy of alternative Fregean or Kripkean approaches in analyzing such issues about representation see Jenefer Robinson, "Two Theories of Representation," Synthese vol. 12 (1978): 37-53.

8. For evidence that one can perceive representational content, see my article "The Perception of Representational Content." 281-97.

9. Ruth Millikan, "Biosemantics," The Journal of Philosophy vol. 86 (1989):

10. E.g., Willard Van Orman Quine, Word and Object, (Cambridge, Mass.: The M.I.T. Press, 1960), sec. 41.

11. Sometimes called a "thisness," e.g. by Robert M. Adams in "Primitive Thisness and Primitive Identity," The Journal of Philosophy vol. 76 (1979): 5-26. For arguments that a thisness cannot be analyzed in purely general terms see Thomas Hofweber, "Supervenience and Object-dependent Properties," The Journal of Philosophy vol. 102 (2005): 5-32. This non-modal concept of a thisness must be distinguished from the modal concept of existence in different possible worlds with different properties, e.g., as discussed in David Kaplan, "How to Russell a FregeChurch," The Journal of Philosophy vol. 72 (1975): 716-29.

12. Including any of the non-comprehensive kinds of information repd by reps of $X$. As evidence of this, perception of $X$ itself might be in circumstances in which the information derivable from $X$ was no more than that derivable from typical reps of $X$.

13. Though the fundamentality of the distinction has been questioned, originally by Frank Ramsey in "Universals," Mind vol. 34 (1925): 401-17, and more recently by Fraser MacBride, e.g., in "The Particular-Universal Distinction: A Dogma of Metaphysics?” Mind vol. 114 (2005): 565-614.

14. For other criticisms of resemblance theories of representation see, e.g., Dominic Lopes Understanding Pictures (New York: Oxford University Press, 1996), and my book The Double Content of Art. 
15. For a broader discussion of problems involving informational indeterminacy issues, see my forthcoming article "Representationalism and Indeterminate Perceptual Content" in Phenomenology and the Cognitive Sciences.

16. Robert Cummins and Pierre Poirier, "Representation and Indication," in Hugh Clapin, Phillip Staines and Peter Slezak, eds., Representation in Mind (New York: Elsevier 2004), 21-40.

17. For evidence and further discussions, see my articles "The Double Content of Perception," Synthese vol. 146, no. 3 (September 2005): pp. 224-243; "The Twofold Orientational Structure of Perception," Philosophical Psychology vol. 18, no. 2 (April 2005): 187-203; and "Representationalism and Indeterminate Perceptual Content."

18. See sec. 3 of the RT article "A Representationalist Theory of Generality" and my book The Double Content of Art.

19. Also discussed in The Double Content of Art and in my article "Perceptual Causality Problems Reflexively Resolved," Acta Analytica vol. 20 (Summer 2005): $11-31$.

20. Ruth Millikan, On Clear and Confused Ideas, chap. 6, and Varieties of Meaning (Cambridge, Mass.: The MIT Press 2004), chap. 9.

21. E.g., see Drew Khlentzos, Naturalistic Realism and the Antirealist Challenge (Cambridge, Mass.: The MIT Press 2004) for a recent summary.

22. For a survey of relevant issues see Henry Krips, "Measurement in Quantum Theory," in Edward N. Zalta, ed., The Stanford Encyclopedia of Philosophy (Winter 1999 Edition), http://plato.stanford.edu/archives/win 1999/entries/qt-measurement/.

23. E.g., Fred Dretske, Knowledge and the Flow of Information (Cambridge, Mass.: The MIT Press, 1984), and Jerry Fodor, A Theory of Content and Other Essays (Cambridge, Mass.: The MIT Press, 1990). 